\title{
Reacciones leprosas
}

\author{
Leprosy reactions
}

\section{Carolina Hoyos-Gómez', Nora Cardona-Castro ${ }^{2 \Xi C v L A C}$}

Fecha correspondencia:

Recibido: junio 4 de 2015.

Revisado: septiembre 11 de 2015.

Aceptado: abril 19 de 2016.

Forma de citar:

Hoyos-Gómez C, Cardona-Castro

N. Reacciones leprosas. Rev CES

Med 2016; 30(2): 200-209.

\author{
Open access \\ (C) Copyright \\ Licencia creative commons \\ Ética de publicaciones \\ Revisión por pares \\ Gestión por Open Journal System \\ ISSN 0120-8705 \\ e-ISSN 2215-9177
}

Sobre los autores:

1. Estudiante de Medicina

Universidad CES.

2 MD, MSc, PhD. Investigadora

Instituto Colombiano de Medicina

Tropical y Facultad de Medicina.

Universidad CES.

Comparte

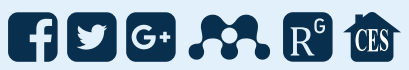

\section{Resumen}

Entre $30-50 \%$ de pacientes con lepra pueden llegar a presentar reacciones inmunológicas, que son eventos agudos que interrumpen el curso crónico de la lepra y son importantes debido a que pueden causar a largo plazo secuelas graves, como el daño permanente de nervios, lo que conlleva a discapacidad y deformidad. Se revisan los diferentes aspectos clínicos y terapéuticos de las reacciones lepromatosas con el fin de profundizar en el conocimiento actual de estas complicaciones de difícil diagnóstico y que son prioritarias para prevenir discapacidades.

Palabras claves: Lepra, Mycobacterium leprae, Reacciones lepróticas, Fenómeno de Lucio.

\section{Abstract}

Between $30-50 \%$ of patients with leprosy could have immune reactions, which are acute events that disrupt the chronic course of leprosy and are important because they can cause serious long-term consequences such as permanent damage nerve, leading to deformity and disability. We review the different clinical and therapeutic aspects of lepromatous reactions in order to deepen the current knowledge of these complications of a difficult diagnosis and are the priority to prevent disabilities.

Keywords: Lepra, Mycobacterium leprae, Leprosy reactions, Lucio's phenomenon.

\section{Introducción}

La lepra, enfermedad infecciosa granulomatosa crónica, es causada por Mycobacterium leprae (bacilo intracelular obligado) (1). A nivel mundial en 2012 se reportaron 181941 casos (prevalencia 0,34 casos/10 000 habitantes) (2), siendo Brasil, India, México y Filipinas los países con más alta prevalencia (3). En Colombia, la prevalencia es menor de 1/10 000; sin embargo, departamentos como Arauca, Norte de Santander, Huila y San-

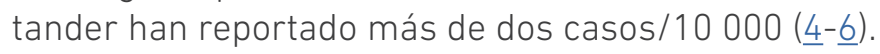

Según Ridley y Jopling existen dos formas clínicas polares, el polo hiperérgico con menos de cinco lesiones cutáneas y mayor compromiso neurítico, con la lepra tuberculoide (LT), y el polo anérgico con la lepra lepromatosa (LL) con múltiples lesiones. Las formas intermedias o dimorfas se subdividen en bordeline tuberculoide (BT), borderline-borderline (BB) y borderline lepromatosa (BL). Los pacientes borderline presentan una o varias máculas hipocrómicas descritas como "queso suizo", situadas con mayor 
Las lepro-reacciones aumentan la morbilidad y son emergencias médicas debido al compromiso neural severo. El diagnóstico y manejo adecuado son necesarios, puesto que pueden confundirse como fallas terapéuticas, y como consecuencia el tratamiento puede ser suspendido o reiniciado, trayendo consecuencias. frecuencia en extremidades, cara y glúteos afección nerviosa, que moderada que pueden evolucionar hacia alguno de los polos $(\underline{4}, \underline{7})$.

La OMS la clasificó en 1981 con base en índice bacilar (IB) y número de lesiones cutáneas, la paucibacilar (PB) con $\mathrm{IB}=0$ y menos de cinco lesiones y la multibacilar (MB), IB>0 y más de cinco lesiones $(\underline{6}, \underline{8})$.

Las lepro-reacciones son eventos agudos con síntomas cutáneos y sistémicos que interrumpen el curso crónico de la enfermedad. Se clasifican como tipo 1 o 2, y una variación del tipo 2 denominada fenómeno de Lucio; aquellos pacientes con índice bacilar mayor o igual a dos y más de dos tercios de superficie corporal comprometidos, tienen mayor riesgo de eventos reaccionales (9). Las reacciones pueden presentarse años post- tratamiento, consecuencia de persistente carga bacilar o componentes antigénicos bacterianos en tejidos (10).

Las lepro-reacciones aumentan la morbilidad y son emergencias médicas debido al compromiso neural severo (13). El diagnóstico y manejo adecuado son necesarios, puesto que pueden confundirse como fallas terapéuticas, y como consecuencia el tratamiento puede ser suspendido o reiniciado, trayendo consecuencias $(\underline{4}, 7)$.

Las lepro-reacciones pueden ocurrir antes o en cualquier momento del tratamiento, estar asociadas a eventos fisiológicos o de riesgo, como infecciones, embarazo, inmunosupresión, cirugía, vacunación y estrés $(\underline{7}, \underline{8}, \underline{12})$.

\section{Reacción tipo 1 o reversa}

Respuesta de hipersensibibilidad tipo IV, exacerbación de la respuesta inmune celular que afecta hasta un tercio de los pacientes; frecuente en bordeline tuberculoide o borderline-borderline, puede ocurrir en borderline lepromatosa o lepra lepromatosa, principalmente durante los seis primeros meses de poliquimioterapia $(\underline{9}, 10,13)$. En pacientes coinfectados con VIH y terapia antirretroviral altamente eficaz, puede presentarse síndrome de reconstitución inmune, caracterizado por mejoramiento de la respuesta inmune celular, ocasionando una reacción inflamatoria semejante a una de reversión, como consecuencia de la destrucción de las células infectadas por el bacilo $(\underline{14}, \underline{15})$.

Las células de Schwann expresan receptores TLR2 (del inglés, Toll like receptors) que se activan en contacto con el antígeno GLP-1 (glicolípido fenólico 1) de M. leprae liberando IL-12 y desencadenando la respuesta inmune celular exacerbada (Th1) contra bacilos intracelulares. Se genera entonces una activación de linfocitos T CD4+ que lleva a la liberación de interleucina-2 (IL-2), interferón gamma (INF $\delta$ ) y factor de necrosis tumoral (TNF-alfa), especies reactivas de oxígeno y otros metabolitos que destruyen la célula de Schwann, produciendo reacción inflamatoria local que produce neuritis y exacerbación de las lesiones (16-26).

Las lesiones cutáneas se tornan eritematosas, edematosas y con superficie lisa y brillante, posteriormente tienden a descamar y ulcerarse. Pueden aparecer nuevas lesiones inflamatorias, edema de cara y miembros superiores e inferiores. Pueden presentarse síntomas sistémicos como fiebre, malestar y anorexia, pero son inusuales (2) (fotografías 1- 1 ). La neuritis es el signo clínico más importante, con dolor, sensibilidad o parestesias y compromiso motor en uno o más nervios. También puede surgir como una neuritis silenciosa, disfunción motora y sensitiva sin dolor (9). Compromete principalmente el cubital, mediano, tibial posterior, peroneo 
común, radial y facial, con secuelas como parálisis facial, anestesia, mano en garra o pie caído (17).

El diagnóstico se hace con el examen físico neurológico y debe apoyarse con biopsia de piel, que reporta edema en granulomas de células epiteloides, edema dérmico y células plasmáticas (16).

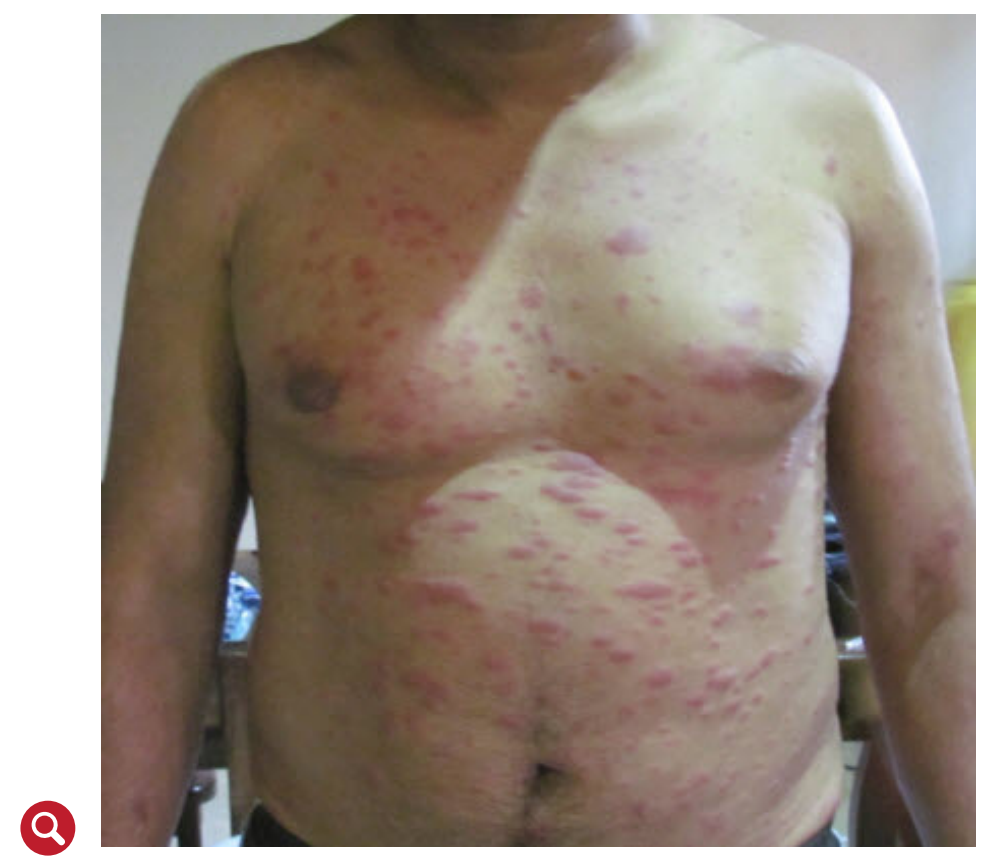

Fotografía 1. Reacción tipo 1. Múltiples lesiones eritematosas

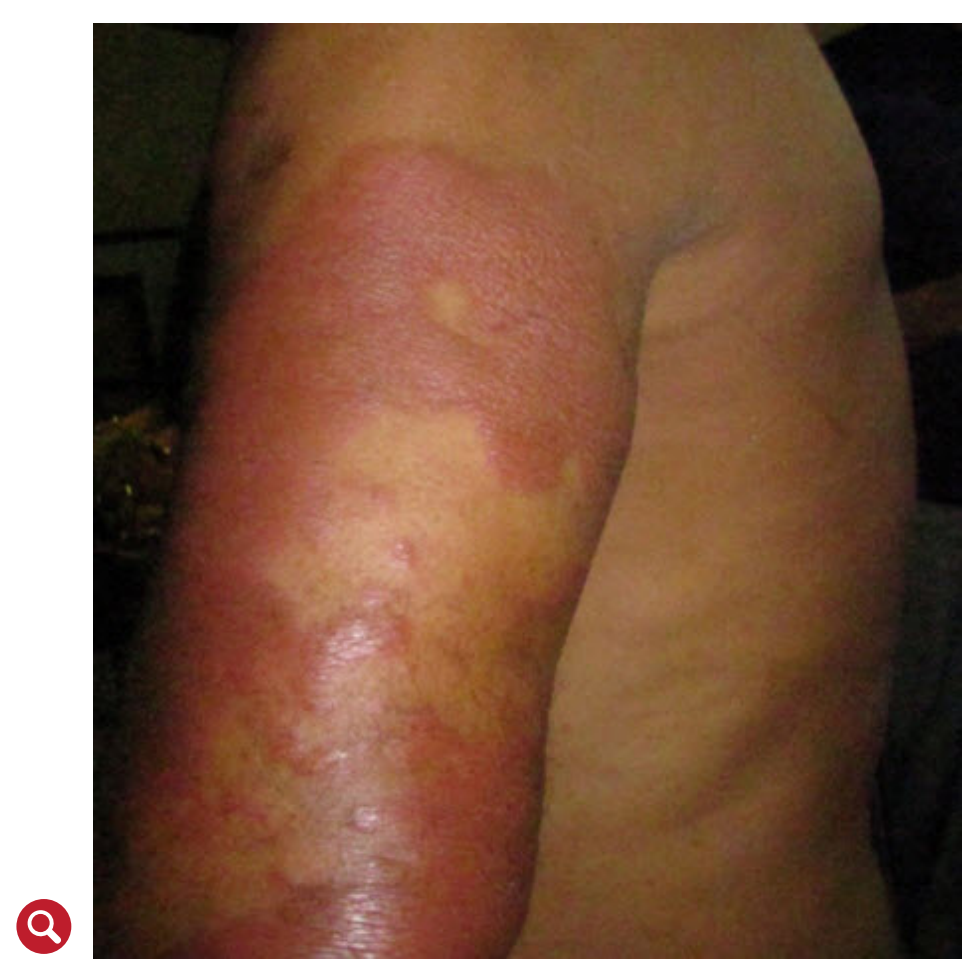

Fotografía 2. Reacción tipo 1. Placas eritematosas descamativas de miembro superior izquierdo 
El tratamiento busca disminuir la inflamación aguda, controlar el dolor y revertir el daño nervioso. Si los síntomas son leves se recomienda aspirina 0 acetaminofén, pero si son severos es pertinente el uso de corticoesteroides solos o asociados a talidomida.

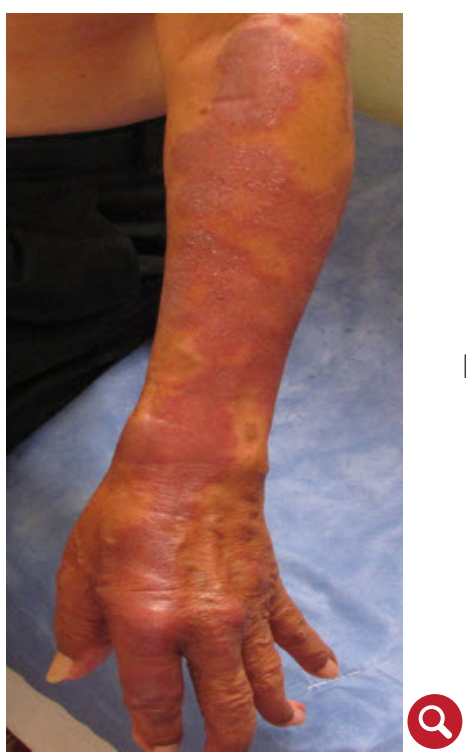

Fotografía 3. Reacción tipo 1. Placas edematosas y eritematosas descamativas de miembro superior izquierdo
Existen dos tipos de reacciones tipo 1: up-grading y down-grading, definidas por la modificación de la inmunidad celular, lo cual puede llevar al paciente a presentar menos bacterias por mejoría inmunológica con manifestaciones clínicas severas (reversión o up-grading), o con mayor número de bacterias debido a un empeoramiento inmunológico con una forma de presentación clínica menos severa (down-grading). Es por ello que a pesar de que la sospecha diagnóstica sea clínica debe hacerse la biopsia de manera obligatoria para diferenciar estos dos tipos de fenómenos reaccionales tipo $1(\underline{7}, \underline{17})$.

El tratamiento busca disminuir la inflamación aguda, controlar el dolor y revertir el daño nervioso. Si los síntomas son leves se recomienda aspirina o acetaminofén, pero si son severos es pertinente el uso de corticoesteroides solos o asociados a talidomida $(\underline{13}, \underline{26})$. Se recomienda evaluar los síntomas cada dos semanas con pruebas de sensibilidad y motoras, así como la inmovilización de extremidades afectadas y posteriormente la rehabilitación $(\underline{3}, 17)$ (cuadro 1).

Cuadro 1. Características de las lepro-reacciones (4-17)

\begin{tabular}{|c|c|c|c|}
\hline $\begin{array}{l}\text { Reacciones } \\
\text { Inmunidad }\end{array}$ & $\begin{array}{l}\text { Tipo } 1 \\
\text { Celular }\end{array}$ & $\begin{array}{c}\text { Tipo } 2 \\
\text { Humoral }\end{array}$ & $\begin{array}{l}\text { Fenómeno de Lucio } \\
\text { Humoral }\end{array}$ \\
\hline Presentación & Se presenta más en TT, BT y BB & Se presenta más en $B L$ y LL & LL o LLD \\
\hline Tiempo de presentación & $\begin{array}{l}\text { Más frecuente a los seis meses } \\
\text { de tratamiento, pero también } \\
\text { antes o posterior a este }\end{array}$ & $\begin{array}{l}\text { Antes, durante o después del } \\
\text { tratamiento }\end{array}$ & $\begin{array}{l}\text { Antes, durante o después del } \\
\text { tratamiento }\end{array}$ \\
\hline Clínica & $\begin{array}{l}\text { Exacerbación de las lesiones } \\
\text { cutáneas previas, nuevas } \\
\text { lesiones y neuritis }\end{array}$ & $\begin{array}{l}\text { Nódulos subcutáneos dolorosos, } \\
\text { neuritis, iritis, episcleritis, } \\
\text { orquitis. Glomerulonefritis por } \\
\text { inmunocomplejos. }\end{array}$ & $\begin{array}{l}\text { Placas eritematosas que se } \\
\text { vuelven ampollas, se necrosan } \\
\text { y se ulceran. Dejan cicatrices } \\
\text { atróficas }\end{array}$ \\
\hline Compromiso sistémico & Escaso & $\begin{array}{l}\text { Marcado (fiebre, malestar general, } \\
\text { mialgias, edema, artralgia y } \\
\text { linfadenomegalia) }\end{array}$ & Común \\
\hline
\end{tabular}


La reacción tipo 2 es una vasculitis leucocitoclástica siendo el eritema nodoso leproso la manifestación clínica más frecuente y a veces única expresión de la reacción.
Los pacientes con reacciones severas deben hospitalizarse e iniciarles poliquimioterapia si no han empezado; si está bajo poliquimioterapia no suspenderse (9). El $60 \%$ de pacientes presentan mejoría clínica en doce semanas; sin embargo, se recomienda continuar tratamiento hasta por veinte semanas (13). En quienes las reacciones reversas son recurrentes, mantener prednisolona 5 a $10 \mathrm{mg}$ durante varios meses (4). Aquellos pacientes con menos de seis meses de daños neurales responden mejor a esteroides que aquellos con compromiso de larga data (9).

En pacientes con corticoterapia prolongada, síntomas muy intensos, sin mejoría o difícil control, son útiles los pulsos endovenosos de metilprednisolona aplicados bajo estricta vigilancia y preferiblemente hospitalizados (20).

En pacientes con intolerancia a los corticoesteroides pueden ser eficaces azatriopina, metotrexato y ciclosporina. Azatriopina más prednisolona por ocho semanas es tan eficaz como prednisolona por doce (16). La descompresión quirúrgica del tronco nervioso afectado se recomienda para pacientes que persisten con dolor luego del tratamiento con corticoesteroides (9).

Los pacientes con lepra coinfectados con VIH en tratamiento con corticoterapia tienen una respuesta igual a los pacientes sin inmunosupresión (13).

\section{Reacción tipo 2 o eritema nodoso leproso (ENL)}

Se trata de un cuadro de hipersensibilidad inmune asociado a estado febril agudo que ocurre en el 60-75\% de pacientes con lepra MB, $10 \%$ BL y 20-47 \% LL. Más frecuente en la segunda década de la vida y durante el primer año de poliquimioterapia, pero puede ocurrir antes o posterior al tratamiento, afecta igual a ambos sexos; con frecuencia es crónica con 2,6 episodios recurrentes en promedio (27). La forma grave y de mayor duración se presenta con poliquimioterapia de doce meses en lugar de veinticuatro $(\underline{11}, \underline{21}, 22)$.

Es mas frecuente en pacientes con índice bacilar mayor o igual a dos, con más de cinco troncos nerviosos engrosados y lesiones cutáneas, infiltración difusa y anticuerpos IgM GLP-1 positivos (17). Es un evento agudo con reacción de células Th2 que promueven la expresión de IL4, IL5 e IL10, que activa la respuesta inmune humoral que produce inmunocomplejos -anticuerpos dirigidos a antígenos de $M$. leprae antigenos circulantes de M. que se depositan especialmente en dermis e hipodermis-, hay activación del complemento y atracción de granulocitos generando daño al tejido afectado. Se describe una tendencia en la recuperación de la respuesta inmune celular con un aumento del TNF-alfa e INF-gamma que conllevan a la destrucción del bacilo $(\underline{17}, \underline{27})$.

La reacción tipo 2 es una vasculitis leucocitoclástica siendo el eritema nodoso leproso la manifestación clínica más frecuente y a veces única expresión de la reacción; se caracteriza por pápulas con nódulos eritematosos de 2-5 mm de diámetro, dolorosos y calientes, superficiales o profundos, más palpables que visuales y pueden ulcerarse, se localizan mayormente en la superficie extensora de extremidades y cara. Si estos no desaparecen se desarrolla una paniculitis crónica dolorosa que persiste a veces durante meses o años, asociada a síntomas generales (cuadro 1) (Fotografía 4) (17,19). 


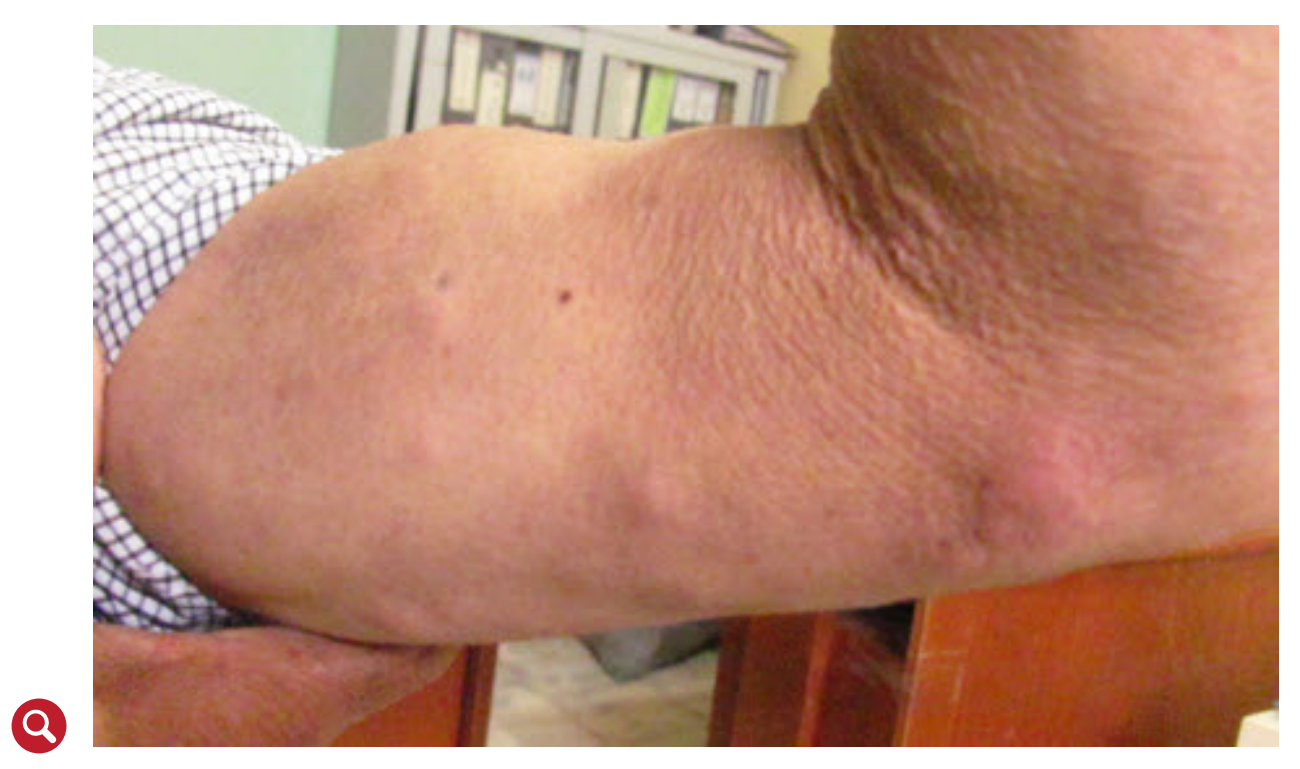

Fotografía 4. Eritema nodoso leproso o reacción tipo 2

Otras manifestaciones son neuritis, dolor, edema en miembros superiores e inferiores menos intensos que en la reacción tipo 1, orquitis, iritis, episcleritis y epistaxis (27). Se puede encontrar hepatoesplenomegalia y proteinuria transitoria por glomerulonefritis debido a amiloidosis o depósitos de inmunocomplejos (6-50 \% de pacientes). Los tipos de glomerulonefritis más comunes son la endocapilar proliferativa, la mesangioproliferativa y la membranoproliferativa $(\underline{18}, 22)$.

En el tratamiento se debe controlar la inflamación aguda, disminuir el dolor y revertir el daño nervioso, continuar la poliquimioterapia y adicionar antiinflamatorios.
Algunos autores consideran al eritema polimorfo leproso como variante clínica de la lepro-reacción tipo 2, con manchas o placas con ampolla central, bordes elevados, inflamados y eritematosos que pueden ulcerarse por su parte central, principalmente en cara, cuello y región escapular (29).

Se realiza diagnóstico clínico que se confirma con biopsia la cual muestra paniculitis lobular con infiltrado inflamatorio de polimorfonucleares en dermis e hipodermis y bacilos ácido alcohol resistentes (BAAR) al interior de los macrófagos (20).

En el tratamiento se debe controlar la inflamación aguda, disminuir el dolor y revertir el daño nervioso, continuar la poliquimioterapia y adicionar antiinflamatorios (9). ELN se clasifica leve, moderado o severo. Leve cuando tiene hasta diez lesiones cutáneas sin síntomas sistémicos; moderado entre diez y veinte lesiones cutáneas sin síntomas sistémicos o severo con más de veinte lesiones cutáneas y compromiso sistémico (cuadro 1) (24). Los casos leves se tratan con antipiréticos y analgésicos; los moderados con talidomida, solo en hombres, mujeres postmenopáusicas o que usen métodos anticonceptivos, debido al alto riesgo de teratogenicidad $(\underline{24}, 25)$. En complicaciones se debe adicionar prednisona a dosis altas por periodo prolongado (22). En casos severos se recomienda manejo intrahospitalario con talidomida, si se asocia a alguna complicación, iniciar esteroides. Cuando está contraindicada la talidomida se utiliza prednisona con clofazimina. Esta última también en pacientes con lepro-reacción tipo 2 a repetición (21).

Las indicaciones para el uso esteroides mas talidomida, son: compromiso de troncos nerviosos, edema inflamatorio en extremidades, lesiones oculares como iritis o iridociclitis, glomerulonefritis, orquiepididimitis, artritis y fenómeno de Lucio $(\underline{21}, 24)$. 
Fenómeno de Lucio Vasculitis necrotizante en vasos de mediano calibre considerada como fenómeno reaccional tipo 2 y ocurre en lepra bonita o lepromatosa nodular.
Los esteroides se utilizan hasta la mejoría clínica, promedio de tres a cinco meses, dosis inicial de quince a veinte días y luego se empieza su desmonte. Paralelamente se prescribe tiabendazol $25-50 \mathrm{mg} / \mathrm{kg} /$ día/dos días, o albendazol 400 mg/día/3 días, debido al riesgo de hiperinfección por Strongyloides stercoralis (14,24).

Se recomienda infliximab como complemento a esteroides para evitar complicaciones por infecciones oportunistas (23).

Existe mortalidad asociada: una cohorte de pacientes etíopes reportó 8 de 99 pacientes muertos por complicaciones atribuidas a uso crónico de corticosteroides $(\underline{28})$.

\section{Fenómeno de Lucio}

Vasculitis necrotizante en vasos de mediano calibre considerada como fenómeno reaccional tipo 2 y ocurre en lepra bonita o lepromatosa nodular. Es la forma anérgica de la enfermedad, presenta infiltración difusa de piel, madarosis, alopecia corporal, anhidrosis y compromiso de mucosas nasal y laríngea (22). Se ha reportado con mayor frecuencia en Centroamérica, pero también en Europa y Asia (19).

Estudios recientes implican a Mycobacterium lepromatosis como causa del fenómeno de Lucio y de formas seronecróticas severas; hay reportes en México, Canadá y Singapur. Se ha reportado que M. lepromatosis y M. leprae pueden causar infección mixta en un mismo paciente $(2,22)$.

Inicia con máculas violáceas dolorosas o placas rodeadas de eritema que forman infartos hemorrágicos formando ampollas que se ulceran, se necrotizan dejando cicatrices atróficas, más comunes en miembros inferiores, antebrazos y glúteos, duran tres a cuatro semanas y pueden encontrarse en diferente evolución $(\underline{2}, 19)$. La hepatoesplenomegalia, iridociclitis, orquitis, epididimitis y agravamiento de las neuritis pueden presentarse (17) (

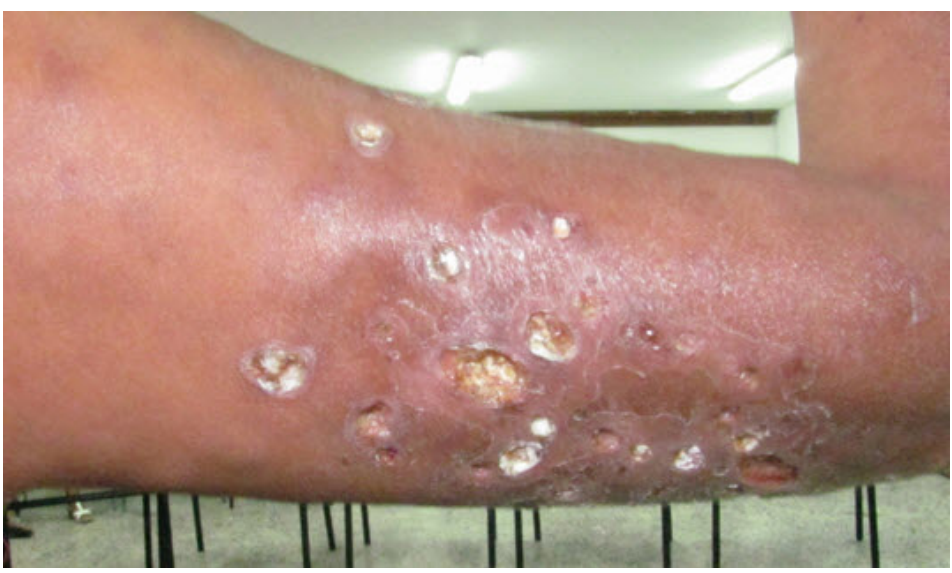

Q Fotografía 5. Eritema nodoso necrotizante o fenómeno de Lucio

El diagnóstico es clínico y la biopsia lo confirma, con necrosis epidérmica por oclusión de vasos sanguíneos subcapilares, escasos neutrófilos y abundantes BAAR $(\underline{2}, 19)$. La patogénesis es poco clara, aunque se demostró IgG y componentes del complemento (C3) en paredes de vasos sanguíneos de mediano calibre de la dermis, así como inmunocomplejos circulantes (19). 
Iniciar poliquimioterapia si no se ha hecho y talidomida y esteroides; también se ha documentado tratamiento con rifampicina a altas dosis (600 mg/día) hasta el control de la reacción o plasmaféresis en pacientes que no remiten al manejo $(\underline{7}, \underline{25})$ (cuadro 1).

\section{Conclusiones}

El 30-50 \% de los pacientes con lepra pueden desarrollar complicaciones inflamatorias agudas, no necesariamente consecuencia de la poliquimioterapia. Se asocian a exacerbación o aparición de nuevas lesiones cutáneas, edema y dolor en nervios afectados con pérdida de funciones sensitivas y motoras, deformidad e incapacidad. Los objetivos principales para evitar secuelas son la detección y manejo tempranos.

\section{Agradecimientos}

Al Dr. Héctor Serrano por las fotografías. Colciencias código 325656933516.

\section{Bibliografía}

1. Sandoval R, Rada M E, Borges R, Aranzazu N. Eritema nodoso leproso en Hansen: Caracteristicas clínicas y respuesta de anticuerpos frente a la proteina LID-1. Dermatol Venez 2013;1-13. http://revista.svderma.org/index.php/ojs/article/ view $/ 527$

2. Lastória JC, Abreu A MM. Leprosy: review of the epidemiological, clinical, and etiopathogenic aspects - Part 1. Anais Brasileiros de Dermatologia. 2014 Apr;89(2):218. https://www.ncbi.nlm.nih.gov/pmc/articles/PMC4008049/

3. Kamath S, Vaccaro SA, Rea TH, Ochoa MT. Recognizing and managing the immunologic reactions in leprosy. Journal of the American Academy of Dermatology 2014;71(4):795-803. http://europepmc.org/abstract/med/24767732

4. Colombia, Ministerio de salud y protección social, Organización Paramericana de la Salud. Guía de atención integral de la lepra. 2012.

5. Cardona-Castro N. Leprosy in Colombia: post elimination stage? Leprosy Review. 2013;84(3):247. http://www.lepra.org.uk/platforms/lepra/files/lr/Sept13/1843. $\underline{\mathrm{pdf}}$

6. Suzuki Koichi, Akama Takeshi, Akira Kawashima, Yoshihara Aya, Yotsu Rie R., Ishii Norihisa. Current status of leprosy: epidemiology, basic science and clinical perspectives. The Journal Of Dermatology. 2012;39(2):129. http://onlinelibrary. wiley.com/doi/10.1111/j.1346-8138.2011.01370.x/abstract

7. Lee Delphine J, Rea Thomas H, Modlin Robert L. Leprosy. Fitzpatrick's dermatology in general medicine. Eight edition. New York, NY: McGraw Hill; p. Chapter 186. https://www.bookdepository.com/Fitzpatricks-Dermatology-General-Medicine-Lowell-Goldsmith/9780071669047

8. Anderson H, Stryjewska B, Boyanton BL, Schwartz Mary R. Hansen disease in the United States in the 21st century: a review of the literature. Archives Of Pathology \& Laboratory Medicine. 2007;131(6):986. https://www.researchgate.net/ publication/6286967 Hansen disease in the United States in the 21st Century A review of the literature 
9. Nery JA da C, Bernardes Filho F, Quintanilha J, Machado AM, Oliveira S de SC, Sales AM. Understanding the type 1 reactional state for early diagnosis and treatment: a way to avoid disability in leprosy. Anais Brasileiros de Dermatologia. 2013;88(5):792. https://www.ncbi.nlm.nih.gov/pmc/articles/PMC3798356/

10. Sinésio Talhari, René Garrido Neves, Gerson Oliviera Penna, Maria Leide VanDel-Rey de Oliveira. Hanseníase. cuarta. 2006.

11. Graham AR, Furlong SR, Margoles LM, Owusu KR, Franco-Paredes CM. Clinical management of leprosy reactions. Infectious Diseases In Clinical Practice. 2010;18(4):238. http://journals.lww.com/infectdis/Abstract/2010/07000/Clinical Management of Leprosy Reactions.4.aspx

12. Scollard David M, Martelli Celina M., Stefani MMA, Maroja M de F, Villahermosa L, Pardillo F, et al. Risk factors for leprosy reactions in three endemic countries. The American Journal Of Tropical Medicine And Hygiene. 2015;92(1):114. https:// www.ncbi.nlm.nih.gov/pubmed/25448239

13. Oliveira DT de, Sherlock J, Melo EV de, Rollemberg KCV, Paixao TRS da, Abuawad YG, et al. Clinical variables associated with leprosy reactions and persistence of physical impairment. Revista da Sociedade Brasileira de Medicina Tropical. 2013;46(5):604. http://www.scielo.br/scielo.php?pi$\underline{d}=$ S0037-86822013000500600\&script $=$ sci abstract

14. Talhari C, De Lima Ferreira LC, Ribamar Araujo J, Chrusciak Talhari A, Talhari S. Inmune reconstitution inflammatory syndrome or upgrading Type 1 reaction? Report of two AIDS patients presenting a shifting from borderline lepromatous leprosy to borderline tuberculoid leprosy. Lepr Rev (2008) 79, 429-435. https:// www.lepra.org.uk/platforms/lepra/files/lr/Dec08/Lep429-435.pdf

15. Bhat R, Pinto M, Dandakeri S, Kambil S. Ulcerating type 1 lepra reaction mimicking lazarine leprosy: an unusual presentation of immune reconstitution inflammatory syndrome in an HIV-infected patient. International journal of STD \& AIDS. 2013. http://std.sagepub.com/content/24/12/992.short

16. Walker SL, Lockwood DN. Leprosy type 1 (reversal) reactions and their management. Leprosy review. 2008;79(4):372. https://www.ncbi.nlm.nih.gov/pub$\underline{\mathrm{med} / 19274984}$

17. Guerrero ET, Martínez FV, Diéguez CEA, Arrazola J, Arenas R. Lepra. Clasificación y cuadro clínico. Dermatol Rev Mex. 2012;56(1):47-54. www.medigraphic.com/ pdfs/derrevmex/rmd-2012/rmd121g.pdf

18. Berrío Elier J, Jaramillo F. Lepra lepromatosa, fenómeno de Lucio, paniculitis mixta más vasculitis y daño renal. Acta Médica Colombiana. 1999;24(3). www. actamedicacolombiana.com/anexo/articulos/03-1999-06.pdf

19. Kaur C, Thami GP, Mohan H. Lucio phenomenon and Lucio leprosy. Clinical And Experimental Dermatology. 2005;30(5):527. http://onlinelibrary.wiley.com/ doi/10.1111/j.1365-2230.2005.01860.x/abstract 
20. Ministério da Saúde. Secretaria de Vigilância em Saúde. Departamento de Vigilância Epidemiológica. Orientações para uso corticosteroides em Hanseníase. MS; 2010. http://bvsms.saude.gov.br/bvs/publicacoes/orientacoes para corticosteroides hanseniase.pdf

21. Voorend CGN, Post EB. A Systematic Review on the epidemiological data of erythema nodosum leprosum, a type 2 leprosy reaction. Vinetz JM, editor. PLoS Neglected Tropical Diseases. 2013;7(10):e2440. http://journals.plos.org/ plosntds/article?id=10.1371\%2Fjournal.pntd.0002440

22. Walker SL, Waters MFR, Lockwood DNJ. The role of thalidomide in the management of erythema nodosum leprosum. Leprosy Review. 2007;78(3):197-215. https://www.ncbi.nlm.nih.gov/pubmed/18035771

23. Faber WR, Jensema AJ, Goldschmidt WFM. Treatment of recurrent erythema nodosum leprosum with Infliximab. New England Journal of Medicine. 2006;355(7):739. http://www.nejm.org/doi/full/10.1056/NEJMc052955

24. Sehgal VN. Lucio's phenomenon/erythema necroticans. International Journal Of Dermatology. 2005;44(7):605. https://www.researchgate.net/publication/7758958 Lucio's phenomenonerythema necroticans

25. Gamboa LAo. Lepra. Empresa Social del Estado Centro Dermatológico "Federico Lleras Restrepo"; 2010. http://www.dermatologia.gov.co/?idcategoria=68\&download $=Y$

26. Walker SL, Lockwood DNJ. The clinical and immunological features of leprosy. British Medical Bulletin. 2006;77-78(1):121. http://bmb.oxfordjournals.org/content/77-78/1/103.full

27. Walker SL, Balagon M, Darlong J, Doni SN, Hagge DA, Halwai V, et al. ENLIST 1: An International Multi-centre Cross-sectional Study of the Clinical Features of Erythema Nodosum Leprosum. PLoS Negl Trop Dis. 2015;9(9):e0004065. http:// journals.plos.org/plosntds/article?id=10.1371/journal.pntd.0004065

28. Walker SL, Lebas E, Doni SN, Lockwood DNJ, Lambert SM. The mortality associated with erythema nodosum leprosum in Ethiopia: a retrospective hospital-based study. PLoS Negl Trop Dis. 2014;8(3):e2690. http://journals.plos.org/ plosntds/article?id=10.1371\%2Fjournal.pntd.0002690

29. Moreira Ríos I, Vázquez Rivera C, Cruz Rodriguez E, Rivera Moreira A, Mujica González DB. Reacción en la lepra. Presentación de un caso clínico. Revista de Ciencias Médicas de Pinar del Río. 2012;16(2):232. http://scielo.sld.cu/scielo. php?script=sci abstract\&pid=S1561-31942012000200017 